\title{
Application of Digital Document Center System in Chinese Petroleum Enterprise
}

\author{
Yue Sun \\ 310Room, Offshore Oil Building A, Bohai Oil Road No. 688, Binhai New Area, Tanggu District, \\ Tianjin, China \\ sunyue4@cnooc.com.cn
}

Keywords: Digital document; File management; Information system; Petroleum enterprise

\begin{abstract}
Currently, petroleum enterprises in China handle hundreds of millions of documents and archives every day, most of which are managed in the traditional paper document management and distribution system. This traditional document management model has significantly slowed down the preservation and sharing ability of documents in giant petroleum enterprises across China. In this paper, we analyze the problems of traditional document management model. By the description, establishment and application of digital document system that centers on knowledge management and content management, we achieve a standardized and centralized management of documents and archives in petroleum enterprise. Applications of digital document system eliminates the drawbacks of traditional paper document management system, and provide a feasible solution to reduce cost and improve efficiency of petroleum enterprises in China. The prototype in CNOOC can be a useful reference to other enterprises in China and around the world.
\end{abstract}

\section{Introduction}

Oil, one of the most important energy sources in the world which controls the economic lifeline of a country. In recent years, with the rapid development of Chinese oil industry, domestic oil companies, especially state-owned large oil enterprises which contain CNPC, Sinopec Group and CNOOC also experienced a leap forward development. According to the 2014 Fortune 500 list shows that Sinopec Group and CNDC in 3 and 4, even the relatively small scale of CNOOC also into the top 100, ranked 93.But it is hard to imagine that the three major state-owned oil enterprises which have three million employees, thousands of branches and hundreds of millions of documents every day, is seriously lagging behind in the document management as the international derailment, Not to mention other smaller private oil companies. So far, the most of domestic oil companies still mainly rely on the way of traditional paper-based documents combined with distributed electronic documents in the document management. The higher degree of information of petroleum enterprises is through the LAN, to set up a simple public document server achieve the purpose of management of documents, but the storage path and the form of these documents are not the same, the security cannot be guaranteed, and it is difficult to find. Such the management is very easy to cause the damage, loss, leakage of document and other phenomena, it is not conducive to the retrieval, borrow, transport of document and so on. Therefore, through the establishment of a unified system of digital document center, became one of the most effective methods to solve the above problems. It is the embodiment of content management, through the document specification, reasonable, centralized management, it effectively improve the sharing rate and the use rate of the document, eliminate all sorts of drawbacks of the traditional document management.

\section{Design and Application of Digital Document Center System in Chinese Petroleum Enterprise}

To solve the above problems in the process of document management, the domestic petroleum enterprises can accord to its characteristics and needs, establish digital document center system of their own which can manage their documents by scientific and effective. $t$ present, the domestic enterprise digital document center system, mostly based on existed content management solution, then according to characteristics and needs of them own, develop the solution in second by Java 
Script Technology and so on. In the domestic application of content management, the famous solutions include Opentext's Livelink platform, IBM's FileNet platform. The following FileNet P8 platform of IBM company as an example, show how to establish the digital document system.

Introduction of the Digital Document Center System Development Platform. File Net P8 platform of IBM Company is an excellent enterprise content management solution. It can easily complete the document management of enterprise level by the powerful function of content management of its own. It can store all kinds of structured and unstructured documents, manage a wide range of enterprise content and object, including images, audio, video, electronic documents, reports, web content analysis report and so on. And it a control mechanism for document permissions, can ensure the safety of electronic document.

The basic framework of File Net P8 platform as fig. 1 which consists of four parts which are the Content Engine, Process Engine, Application engine and Rendition Engine. Content Engine mainly manages a wide range of enterprise content and object. Process Engine provides services for all aspects of business process management, such as process execution, process routing, rules management, management of all aspects of simulation and modeling, workflow analysis etc... Rendition Engine can convert different formats of electronic documents to the unfiled format. Application engine is display platform layer. FileNet P8 comes with a Workplace XT application software, can realize the most functions of digital document center system, suitable for small and medium enterprises which do not need the second development. But because it is too rigid, the interface is not friendly and other shortcomings, most oil companies should make full use of the characteristics of FileNet P8 platform, based on the second development for the formation of the final application interface

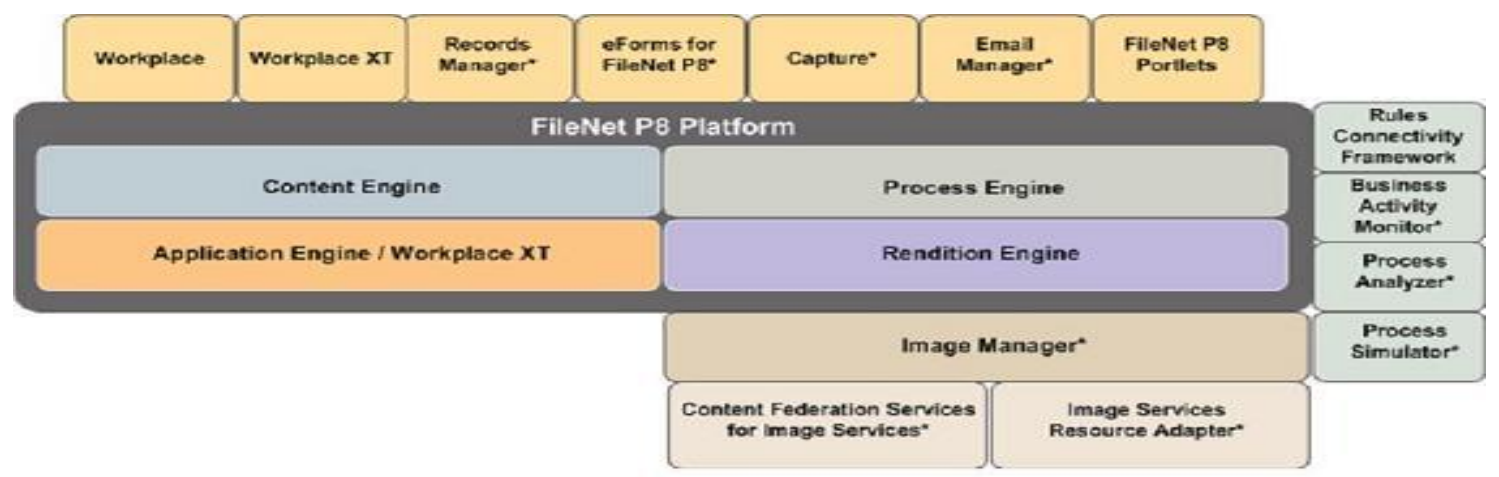

Figure 1. The basic framework of File Net P8

Hardware Structure of the Digital Document Center System. In order to guarantee the high availability of the digital document center system, and give full consideration to the large state-owned petroleum enterprise which have a huge number of electronic document and amount of concurrent using. In the design of the hardware structure should be considered using real application cluster technology and load balancing technology to enhance the stability of the system. At the same time, if the property permits, we can use virtualization technology, as part of the virtual machine to act as a server to improve management efficiency and reduce the cost. The following is general hardware architecture: 


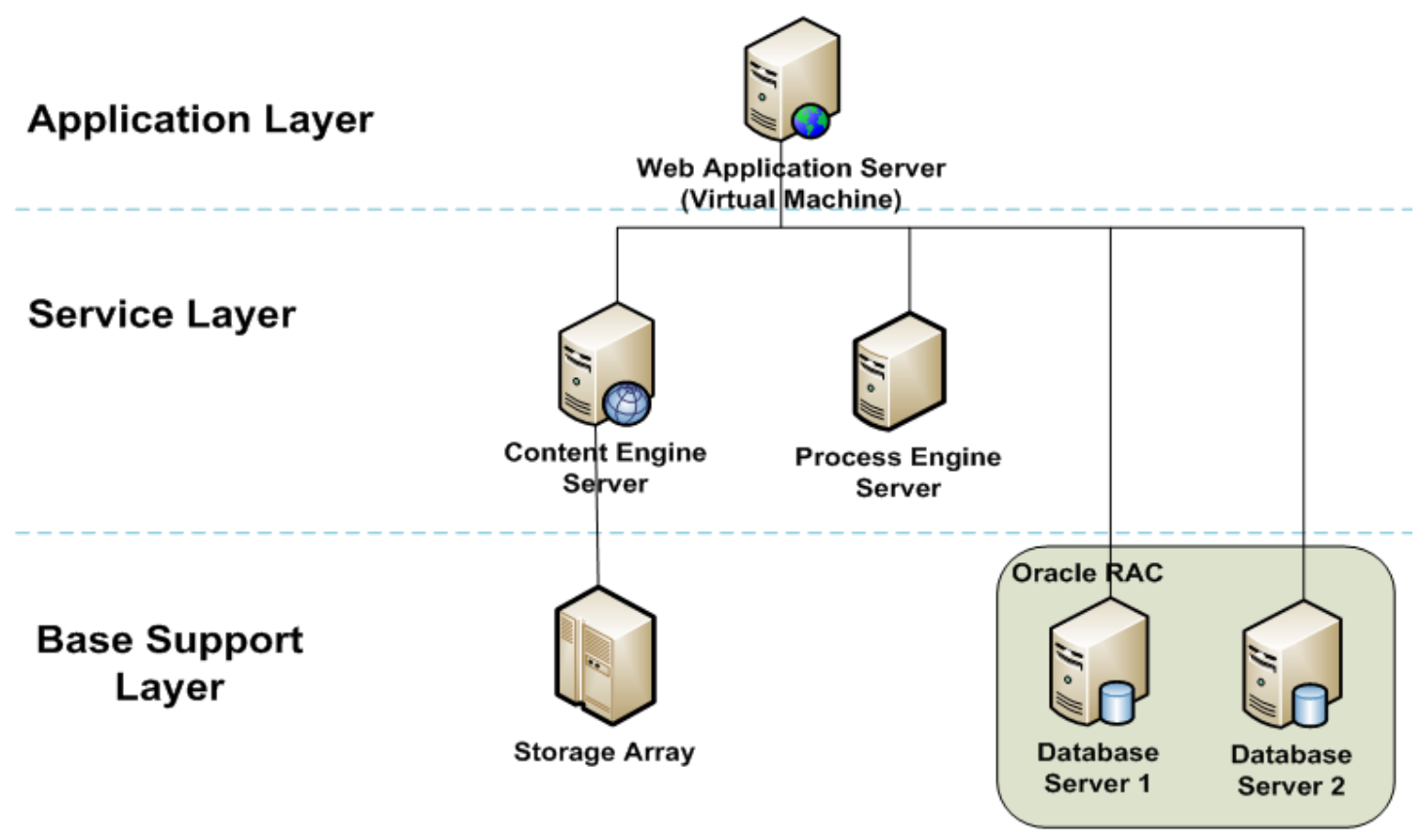

Figure 2. A digital document center system hardware structure

As shown in Fig. 2, the structure consists of three sets of engine and application servers and two sets of database servers. Among them, three engines and application server is a web application server, a content server engine and a process engine server. In order to ensure the speed and the stability of the system, the servers are used IBM's x3850 M2 minicomputer. Web Application Server is used for carrying FileNet P8 Application Engine which publish the front-end application of digital document center system, it can used the virtual machine; Content Engine Server is mainly used to carry the FileNet P8 content engine which store and manage a wide range of enterprise content and object, for example electronic documents, video, image. Therefore, it need larger storage capacity which can be make a storage array expand; Process Engine Server is mainly used to carry the FileNet P8 process engine which provide service and support for the file access and document delivering process. Two Database Server use the Oracle database, hardware is two sets of AIX P5 minicomputers, and use the real application cluster technology. When a database server fails, it can automatically take over from another server so that improve the availability and stability of the system.

Design and Application of Digital Document Center System. According to the size and structure of the petroleum enterprise, you can choose two ways to build a digital document center system. One is put a enterprise as a unit to establish a digital document center system, and this method is suitable for small and medium-sized petroleum enterprises; the other is put the branch of the enterprise as a unit to establish it which regularly upload or other ways to save and unified digital documents to the enterprise, this way is suitable for large state-owned Petroleum Enterprises. Digital document center system built by FileNet P8 can also be integrated with Active Directory which can provide the single sign technology to remove the username and password input, and quickly read the user information in the landing at the same time so that easy to access control.

Function Description. The basic function of the digital document center system should have electronic document upload and download, delete, edit and modify permissions settings etc... In addition, for the convenience of users and management of electronic documents, it is included to create a link, check in and checkout, move the file or folder, document sharing, attribute entry and modification, audit functions etc... Following a brief introduction to some common functions:

(1) Basic function: It can upload, download and delete all kinds of electronic documents, can edit the Word, PowerPoint, documents and pictures online, and can flexibly set the permissions of the electronic documents stored in all.

(2) Create a link: All the file or folder stored can generate a specific link, we can access to the file 
or folder directly, and we can send this link to others by email and copy. If the other has the authority, they can quickly operate target file or folder just click on the link.

(3) Check in and check out: First download the target file by check out function, then upload the file to original path after modify the file. At this time, the file will automatically upgrade, the original and new version are stored in the system. This function is not only convenient to modify the file, but also effectively control the version.

(4) Move the file or folder; You can move the target files to any path freely. This function conduces to document collation and classification.

(5) Document sharing; When a user who has certain authority to the document which can be shared with other users.

(6) Attribute input and modification; Classify documents by its different of contents, such as logging, geological documents and other documents. Then, set the different attributes according to the different document category, such as set well types, well numbers and so on for the oil well documents; set line number and running distance attributes and so on for the geological documents. In addition, make some attribute must be input, in order to document traceability and retrieval. At the same time make the users which have appropriate authority modify the attribute.

(7) Audit; Users have authority through the function keys can view the document history operation record directly, so that you can know what a user operate the document at a certain time. This is conducive to tracing and traceability when the document occur the problem.

The above is part of the common function of digital document center system. Of course, the user can according to their situation and needs to develop more convenient functions.

\section{Conclusions}

In summary, the establishment and application of digital document center system is one of the most effective methods that reduce costs and improve work efficiency in the petroleum enterprises in China. In the light of globalization, information technology is an important embodiment of enterprise's operation efficiency and management level. In order to improve the level of management and to actively participate in international competition, giant enterprises must enhance their information technology by introducing advanced and scientific management concepts and methods such as knowledge and content management. Central digital document system manages the content and knowledge so that information is propagated clearly. Chinese petroleum enterprises should adapt similar digital systems based on their own needs in project operation and management, so that documents are standardized, managed, and archived efficiently and scientifically.

\section{References}

[1] William J. Carpenter: Getting Started with IBM FileNet P8 Content Manager (Packt Publishing, UK 2011)

[2] Todd R. Groff, Thomas P.Jones: FileNet: A Consultant's Guide to Enterprise Content Management (Taylor \& Francis Ltd, UK 2004)

[3] Q.Q. Yang: Archives Science Bulletin, 2014 No.2, p.58 In Chinese

[4] T.F. Meng, G.X. Ma: China CIO News, 2012 No.8, p.60 In Chinese

[5] L. Liu: Archives Science Bulletin, 2013 No.2, p.33 In Chinese 